HIP - $1999-04 / \mathrm{TH}$

NORDITA-99/7

March 21, 2018

\title{
A variational fit to the lattice energy of two heavy-light mesons
}

\author{
A.M. Green ${ }^{a, c}$, J. Koponen ${ }^{a, d}$, and P. Pennanen ${ }^{b}$, \\ ${ }^{a}$ Department of Physics and Helsinki Institute of Physics, P.O. Box 9, FIN-00014 University \\ of Helsinki, Finland \\ ${ }^{b}$ Nordita, Blegdamsvej 17, 2100 Copenhagen Ø, Denmark
}

\begin{abstract}
Recent lattice calculations on the interaction energy of two heavy-light mesons $\left(Q^{2} \bar{q}^{2}\right)$ in $\mathrm{SU}(3)$ are interpreted in terms of the potential for the corresponding single heavy-light meson $(Q \bar{q})$. This model supports earlier work, with four static quarks $Q^{4}$ in $\mathrm{SU}(2)$, that there is a large overestimate of the binding compared with the lattice data - unless the basic $Q \bar{q}$ potentials are modified by a four-quark form factor.
\end{abstract}

PACS numbers: 24.85.+p, 13.75.Lb, 11.15.Ha, 12.38.Gc

${ }^{c}$ email: anthony.green@helsinki.fi

${ }^{d}$ email: jmkopone@rock.helsinki.fi

${ }^{e}$ email: petrus@hip.fi 


\section{Introduction}

In many-body systems, such as nuclei, involving nucleons interacting through mesons, it seems justified to replace the explicit presence of the mesons by internucleon potentials. These are mainly between pairs of nucleons with three-nucleon potentials, in general, playing a minor role. However, in many-body systems involving quarks interacting through gluons, it is not at all clear whether or not a similar potential approach is meaningful. There are basically two schools of thought. On the one hand, for particle physicists, the use of only two-quark potentials in multiquark systems is not even a discussion point, since they simply believe that it can not be correct due to the non-Abelian nature of the gluon fields that lead to gluon-gluon interactions. In fact, others go even further by saying that the whole concept of potentials is not useful in these systems. In spite of these very basic objections, there is a second school of thought - mainly that of many-body physicists well versed in multi-nucleon problems - that continue to treat multi-quark systems with standard many-body techniques using two-quark potentials. One of the most recent and extensive sets of calculations in this latter approach is to be found in Refs. [1, 2]. These describe meson-meson scattering in terms of four quarks [1] and baryonbaryon scattering in terms of six quarks [2]. In each case two (or three) quarks are confined into a cluster by means of an oscillator potential and then two such clusters interact via two-quark potentials. However, there is no a priori justification for this. Over the last few years attempts have been made to clarify this situation by comparing the exact energies of four-quark systems - as calculated on a lattice - with standard many-body models using only two-quark potentials - see Refs. [3, 国] (and references therein). Four-quark systems were chosen for this comparison for several reasons:

1) Such systems exhibit a feature not present in $q \bar{q}$ and $q q q$ descriptions of mesons and baryons namely - the possibility of there being two two-quark color singlet clusters. This situation arises in meson-meson scattering, where the intercluster distance is not restricted by confinement.

2) For systems with many quarks the ability to perform accurate lattice simulations decreases rapidly with the number of quarks - four being essentially the present day limit.

3) For the four-quark system, many-body models in terms of interquark potentials can be expressed in simple and transparent forms.

In Refs. [3] - [5] this comparison program has already been made with the outcome, shown in Figure 1, that the resulting four-quark binding energies are grossly overestimated by the models, if only standard two-quark potentials are used. This is nothing more than the well known van der Waals effect [6] and, as shown in Refs. [3] - [5], can be overcome by introducing a four-quark form factor containing a single free parameter. In Ref. [4] it was further shown that this single parameter was capable of giving a reasonable understanding of 100 pieces of data - the ground and first excited states of configurations, calculated on a $16^{3} \times 32$ lattice, from different four-quark geometries - rectangles, quadrilaterals and tetrahedra - in addition to the squares of Figure 1. But in all cases the model without the form factor yielded far too much binding for the ground state. This concept of a form factor is a familiar and successful technique when dealing with systems of interacting baryons, where the baryon-baryon potentials due to 
the exchange of well known mesons (e.g. the $\pi$ or $\rho$ ) have their short-range singular behaviors modified by form factors. The form of the latter are usually determined by first fitting basic two-body baryon-baryon data before embarking on the study of multi-nucleon systems. Here the same philosophy is attempted by first determining a four-quark form factor from the four-quark lattice data before embarking on the study of the multi-quark systems involved in a microscopic description of meson-baryon or baryon-baryon scattering. It should be added that also in the context of forces between quarks the idea of introducing many-body interactions to remove the van der Waals problem is not new. For example, it is discussed by the authors of Refs. [7, 8] when they introduce their alternative model - commonly called the "flip-flop" model.

Unfortunately, the comparisons between the lattice data and the proposed model just discussed had several shortcomings:

a) All four quarks were of infinite mass (i.e. the $Q^{4}$ static approximation ).

b) The lattice calculations ignored the possibility for the creation/annihilation of quark pairs (i.e. the quenched approximation).

c) Only two colors were considered for the quarks (i.e. $\mathrm{SU}(2)$ ).

In the present work, the lattice data in Refs. [9]-[11] have these defects partially corrected. Firstly, only two of the four quarks are now static with the other two having masses comparable to that of the strange quark. This $Q^{2} \bar{q}^{2}$ system is often referred to as that of two interacting heavy-light mesons. Secondly, the three color group [SU(3)] of QCD is incorporated. Finally, there is now preliminary lattice data [12] utilising gauge field configurations generated with dynamical fermions 13] i.e. the quenched approximation is no longer necessary. These are major improvements in the lattice work. However, as will be seen later, in the corresponding manybody model some approximations need to be made. But these are not expected to change the model predictions qualitatively. The outcome is that the standard many-body model without a form factor again overestimates the four-quark binding energy. On its own, this result from the $Q^{2} \bar{q}^{2}$ system would not be very convincing. However, when combined with the earlier work on the $Q^{4}$ system, the present result will be seen to clearly support the same conclusion that the use of two-quark potentials alone is not justified. On the positive side, the model also offers an effective interaction - albeit with a four-quark form factor - that has been tuned to fit the $Q^{2} \bar{q}^{2}$ system. The hope is now that this effective interaction is more appropriate than the one without the form factor in other multiquark systems as encountered in meson-baryon or baryonbaryon scattering. Of course, this is only a hope that such an effective interaction is in anyway universal. It can only be substantiated by detailed calculations in other multi-quark systems. At present, our main point is that this effective interaction at least succeeds (by arrangement) in the four-quark case - a feature that is not true with the use of only two-quark potentials.

The above gives a purely theoretical motivation for this work. However, a second motivation is that the $Q^{2} \bar{q}^{2}$ system being studied has many features in common with the interaction between two $B$-mesons, the latter being a combination of a $\bar{b}$-quark and an $u$ or $d$-antiquark. These mesons are now fashionable, since the advent of $B$-meson factories at SLAC and KEK has increased interest in the structure of - and interaction between $-B$-mesons. These facilities will 
not be able to study directly $B B$ reactions. However, the related $B \bar{B}$ system is accessible as a final state of, for example, the decay of the $\Upsilon(4 S, 10580 \mathrm{MeV})$, whose main branching $(\geq 96 \%)$ is into $B \bar{B}$. At present, the experimental emphasis with the $\Upsilon(4 S)$ is the study of the non- $B \bar{B}$ decays - see for example [14].

The following model estimate of the four-quark binding energy (i.e. for $Q^{2} \bar{q}^{2}$ ) is in two parts: a) The energy $E(2)$ of the two separate $Q \bar{q}$ systems.

b) The energy $E(4)$ of the complete four-quark system.

The binding energy $[B(4)]$ of the latter is then defined to be

$$
B(4)=E(4)-2 E(2)
$$

with both $E(2)$ and $E(4)$ requiring separate variational calculations.

In Section 2 the energies of the heavy-light $(Q \bar{q})$ system, extracted from the lattice calculation of Ref. [9], are fitted using a variational solution of the non-relativistic two-body Schroedinger equation. This enables a determination to be made of an effective light quark mass $\left(m_{q}\right)$ that is appropriate for the subsequent non-relativistic four quark studies. In Section 3 the formalism for calculating the binding energy of the four-quark system by a second variational calculation is described and, in Section 4, this is compared with the lattice data of Refs. [9, 11]. Section 5 contains conclusions.

\section{The two-quark system}

In Ref. [9] a new method was introduced for generating quark propagators. For heavy-light $(Q \bar{q})$ mesons this led to a considerable improvement over earlier methods using iterative inversion and enabled estimates to be made of the energies of states with orbital angular momentum $L=0,1,2,3$. In addition, the splitting between the $j=L \pm \frac{1}{2}$ states could also be observed. However, in the present work we are not interested in this later refinement, since we average over spins. This spin-averaged data $E(2, L$, lattice) is now fitted with the non-relativistic Schroedinger equation

$$
[T(2)+V(2)-E(2, L)] \phi=\left[-\frac{d^{2}}{2 m_{q} d r^{2}}+\frac{L(L+1)}{r^{2}}+V(2, r)-E(2, L)\right] \phi=0 .
$$

Here there are two unknowns - the effective quark mass $m_{q}$ and the interquark potential $V(2, r)$. Since the two-quark potential will be an important ingredient in the four-quark model, care must be taken in choosing one that is appropriate in the sense that it is most realistic over that range of $r$ dominant in the four-quark problem. Later it will be seen that it is necessary to perform spacial integrations over $V(2, r)$ and that the integrands are peaked in the range of 2 to 4 lattice spacings. We, therefore, generate a two-quark potential with 40 configurations on a $16^{3} \times 24$ 
lattice at $\beta=5.7$ using two fuzzing levels $(2,13)$. The basic data is given at the on-axis points at $r / a=2, \ldots 9$ and is well fitted $\left(\chi^{2} / \mathrm{dof}=1.65\right)$ by the form (in $\mathrm{fm}^{-1}$ and $r$ in $\mathrm{fm}$ )

$$
a V(2, r)=-\frac{0.309(38)}{r / a}+0.1649(36) r / a+0.629(25) .
$$

This gives a string energy of $(445 \mathrm{MeV})^{2}$ for $a=0.18 \mathrm{fm}$. In Table 1 the lattice data is compared with this fitted potential in Eq. 3. There it is seen that the fit is good over the important range of $r \sim(2-3) a$. In Ref. [15] an alternate form of $V(2, r)$ is given. However, this is designed to get a good fit to the lattice data for large values of $r$ in order to extract an estimate of the string energy, whereas we are more interested in values of $r$ only upto about $5 a$. Using the same form of parametrization as in Eq. 3 their corresponding parameters are 0.2618 (i.e. $\pi / 12$ ), 0.1504 and 0.6674 respectively. This results in a string energy of $(425 \mathrm{MeV})^{2}-$ slightly less than our value. The greatest difference between the two forms is the additive constant, so that at $r=1$ we have $V(2)=-138 \mathrm{MeV}$, whereas they have $-179 \mathrm{MeV}$. This is in spite of the fact that, in both cases, at $r=1$ the original lattice data are well within $1 \%$ of each other. However, it is expected that our potential is more realistic at such small values of $r$, since we introduce an extra degree of freedom by allowing the strength of the coulomb term to be a free parameter - unlike Ref. 15. where it is frozen at $\pi / 12$. Here we only need $V(2, r)$ for the quenched approximation as no fit is performed below to the dynamical fermion data - the reason being that not all the necessary observables have yet been measured.

The other unknown in Eq. 2 is the quark mass $\left(m_{q}\right)$ and this is treated as a free parameter adjusted so that the $E(2, L=0,1,2,3)$ model energies are an average fit to the corresponding lattice data.

An estimate of $E(2, L)$ from Eq. 2 is extracted using the variational principle by simply minimizing the expression

$$
\langle\phi(r)|T(2)+V(2)| \phi(r)\rangle /\langle\phi(r) \mid \phi(r)\rangle
$$

with a variational wavefunction of the form

$$
\phi(r)=\sum_{i=1}^{N_{2}} \beta_{i} \exp \left(-\alpha_{i} r^{2} / 2\right) .
$$

Here the $\alpha_{i}$ and $\beta_{i}$ are the variational parameters, but with $\beta_{1}$ fixed at unity to set the overall normalisation. Later, it will be seen that $N_{2}$, the number of terms in the sum, need not be greater than three to get sufficient accuracy for $E(2, L)$.

The outcome is $m_{q}=400 \mathrm{MeV}$ gives a good overall fit to the data. At this stage no attempt is made to optimize $m_{q}$. However, this value of $m_{q}$ does present a problem, since it is sufficiently small that relativistic effects would be expected to be important. We return to this point later.

The above energies $E(2, L)$, and the four-quark energies calculated in the next section, are obtained using a variational procedure and so are only upperbounds. Therefore, since the 
binding energy defined in Eq. 1 1 can lead to a delicate cancellation between $E(4)$ and $2 E(2)$, it is necessary to know how accurate are the estimates of these separate quantities.

To check the accuracy of $E(2)$, the method described by Eqs. 1 , 5 is used to calculate the energies for the form of the $L$-wave Schroedinger equation treated in Ref. [17]. There the eigenvalues are given to seven significant figures for a series of potentials of the type given in Eq. 3. We find that our variational estimates using Eq. 5 for $N_{2}=2$ or 3 are in agreement with the exact result to about four significant figures - an accuracy much better than is actually needed here.

\section{The four-quark system}

In Ref. [4] a model was developed for understanding the lattice energies of four static quarks $Q\left(\mathbf{r}_{\mathbf{1}}\right) Q\left(\mathbf{r}_{\mathbf{2}}\right) \bar{Q}\left(\mathbf{r}_{\mathbf{3}}\right) \bar{Q}\left(\mathbf{r}_{\mathbf{4}}\right)$ in terms of two-quark potentials. This model, in its simplest form, was constructed in terms of the two basis states that can be made by partitioning the four quarks into two color singlets - namely -

$$
A=[Q(1) \bar{Q}(3)][Q(2) \bar{Q}(4)] \text { and } B=[Q(1) \bar{Q}(4)][Q(2) \bar{Q}(3)],
$$

where $[. .$.$] denotes a color singlet. These two states are not orthogonal and have a normalisation$ matrix of the form - see Ref. [16].

$$
\mathbf{N}(\mathbf{f})=\left(\begin{array}{ll}
1 & \frac{1}{3} f \\
\frac{1}{3} f & 1
\end{array}\right)
$$

In the extreme weak coupling limit the parameter $f=1$ and in the strong coupling limit $f=0$. However, for intermediate situations it is parametrised as

$$
f\left(\mathbf{r}_{1}, \mathbf{r}_{\mathbf{2}}, \mathbf{r}_{\mathbf{3}}, \mathbf{r}_{4}\right)=\exp \left[-b_{s} k_{f}^{S} S\left(\mathbf{r}_{1}, \mathbf{r}_{\mathbf{2}}, \mathbf{r}_{\mathbf{3}}, \mathbf{r}_{4}\right)\right]
$$

where $b_{s}$ is the string energy, $S\left(\mathbf{r}_{1}, \mathbf{r}_{2}, \mathbf{r}_{3}, \mathbf{r}_{4}\right)$ is an area defined by the positions of the quarks and $k_{f}^{S}$ is a free parameter. As discussed in the Introduction, a single value of $\approx 0.5$ for $k_{f}^{S}$ was capable of giving a reasonable understanding of 100 pieces of data - the ground and first excited states of configurations from six different four-quark geometries calculated on a $16^{3} \times 32$ lattice. In this model the interaction between the quarks is expressed as a potential matrix of the form

$$
\mathbf{V}(\mathbf{f})=\left(\begin{array}{ll}
v(13)+v(24) & V_{A B} \\
V_{A B} & v(14)+v(23)
\end{array}\right),
$$

where $V_{A B}=-\frac{f}{3}[v(13)+v(24)+v(14)+v(23)-v(34)-v(12)]$ as expected in the weak coupling limit with the one-gluon-exchange-potential

$$
V=-\frac{1}{3} \sum_{i \leq j} \lambda_{i} \lambda_{j} v_{i j} \text { and } v_{i j}=-\frac{e}{r_{i j}}
$$


Away from the weak coupling limit, $f$ is no longer unity and in addition $v_{i j}$ is taken to be the full two quark potential of Eq. 3. The energy of the four static quarks is then given by diagonalising

$$
\mid \mathbf{V}\left(k_{f}\right)-E\left(4, \text { static }, k_{f}\right) \mathbf{N}\left(k_{f}\right) \mid \psi=0 .
$$

This model, although very simple, contains the same basic assumptions made in the more elaborate many-body models that incorporate kinetic energy e.g. the Resonating Group Method[7]. It is, therefore, reasonable that this simplified model can to some extent check the validity of its more elaborate counterparts.

When only two of the four quarks are static the corresponding matrices for $Q\left(\mathbf{r}_{\mathbf{1}}\right) Q\left(\mathbf{r}_{\mathbf{2}}\right) \bar{q}\left(\mathbf{r}_{\mathbf{3}}\right) \bar{q}\left(\mathbf{r}_{\mathbf{4}}\right)$ can be expressed in a similar form but where the matrix elements are now integrals over the positions of the two light antiquarks. Below we consider basis state $A$ to be the one realised as two separate heavy-light mesons - $[Q(1) \bar{q}(3)]$ and $[Q(2) \bar{q}(4)]$ - when the distance $\mathbf{R}=\mathbf{r}_{\mathbf{1}}-\mathbf{r}_{\mathbf{2}}$ between the two heavy quarks becomes large. In this state the convenient coordinates are then $\mathbf{s}_{1}=\mathbf{r}_{3}-\mathbf{r}_{1}$ and $\mathbf{s}_{2}=\mathbf{r}_{4}-\mathbf{r}_{2}$, whereas for the other partition $B$ the convenient coordinates are $\mathbf{t}_{1}=\mathbf{r}_{3}-\mathbf{r}_{2}=\mathbf{s}_{1}+\mathbf{R}$ and $\mathbf{t}_{2}=\mathbf{r}_{4}-\mathbf{r}_{1}=\mathbf{s}_{2}-\mathbf{R}$. We also use the definition $\mathbf{u}=\mathbf{r}_{\mathbf{3}}-\mathbf{r}_{\mathbf{4}}$.

The variational wavefunction is now taken to have the form

$$
\psi\left(\mathbf{r}_{\mathbf{i}}, f\right)=f^{1 / 2}\left(\mathbf{r}_{\mathbf{1}}, \mathbf{r}_{\mathbf{2}}, \mathbf{r}_{\mathbf{3}}, \mathbf{r}_{\mathbf{4}}\right) \sum_{i=1}^{N_{4}} \exp \left(-\tilde{\mathbf{X}} \mathbf{M}_{i} \mathbf{X}\right),
$$

where $\tilde{\mathbf{X}}=\left(\mathbf{s}_{\mathbf{1}}, \mathbf{s}_{\mathbf{2}}, \mathbf{R}\right)$ and each matrix $\mathbf{M}_{\mathbf{i}}$ has the form

$$
\mathbf{M}_{i}=\frac{1}{2}\left(\begin{array}{ccc}
a_{i} & b_{i} & c_{i} \\
b_{i} & d_{i} & e_{i} \\
c_{i} & e_{i} & g_{i}
\end{array}\right) .
$$

Since the present problem considers the masses of the light quarks to be equal, we in fact use a simplified form of $\mathbf{M}_{\mathbf{i}}$ with $b_{i}=0, d_{i}=a_{i}$ and $e_{i}=c_{i}$. This is not necessary, but it is expected to be the dominant term in such a symmetric case. Already for $N_{4}=2$, this wavefunction is indeed adequate for giving sufficiently accurate four-quark binding energies. Even this choice involves five free parameters $\left(a_{1}, c_{1}, a_{2}, c_{2}, g_{2}\right)$ in the variation - with $g_{1}$ being fixed at unity to set the overall normalisation. In Eq. 13 the parameters $a_{i}$ are analogous to the $\alpha_{i}$ in Eq. 5 and the $g_{i}$ play the role of the $\beta_{i}$. In what follows the positions of the light quarks are integrated over leaving matrix elements that are functions of $\mathbf{R}$. In order to achieve this in any practical way it is necessary to have a form for $f\left(\mathbf{r}_{1}, \mathbf{r}_{2}, \mathbf{r}_{\mathbf{3}}, \mathbf{r}_{\mathbf{4}}\right)$ that has a simpler spatial dependence than the area $S$ used earlier in Eq. 8 for the four static quark case. Here we take the very symmetric form advocated in Ref. [16] - namely -

$$
f=\exp \left[-k_{f} b_{s} \sum_{i \leq j} r_{i j}^{2}\right] .
$$


It should be emphasised that this form of $f$ is purely for numerical simplicity leading to analytical expressions for all matrix elements. Again $k_{f}$ is a free parameter, which should be adjusted to fit the four-quark lattice energies.

The wavefunction in Eq. 12 is used for both states $A$ and $B$. This is an approximation that appears to work well for the $Q^{2} \bar{q}^{2}$ system, since $A$ and $B$ are similar in structure for the $R$ values of interest here.

The normalisation matrix can now be written as

$$
\mathbf{N}^{\prime}\left(R, k_{f}\right)=\left(\begin{array}{ll}
N(R, 0) & \frac{1}{3} N\left(R, k_{f}\right) \\
\frac{1}{3} N\left(R, k_{f}\right) & N(R, 0)
\end{array}\right),
$$

where - after integrating over $\mathbf{s}_{\mathbf{1}}, \mathbf{s}_{\mathbf{2}}-N\left(R, k_{f}\right)$ can be expressed as a sum of terms of the form

$$
\frac{\pi^{3}}{(a X)^{3 / 2}} \exp \left[-\left(Z-\frac{Y^{2}}{X}\right) R^{2}\right]
$$

where $a=0.5\left(a_{i}+a_{j}\right)+3 k_{f}, c=0.5\left(c_{i} \pm c_{j}\right)+2 k_{f}, d=0.5\left(c_{i} \pm c_{j}\right)-2 k_{f}, g=0.5\left(g_{i}+g_{j}\right)+4 k_{f}$, $X=a-k_{f}^{2} / a, Y=c+k_{f} d / a$ and $Z=g-d^{2} / a$.

Since two of the quarks are not static there is now also a kinetic energy matrix

$$
\mathbf{K}^{\prime}\left(R, k_{f}\right)=\left(\begin{array}{ll}
K_{3}(R, 0)+K_{4}(R, 0) & \frac{1}{3}\left[K_{3}\left(R, k_{f}\right)+K_{4}\left(R, k_{f}\right)\right] \\
\frac{1}{3}\left[K_{3}\left(R, k_{f}\right)+K_{4}\left(R, k_{f}\right)\right] & K_{3}(R, 0)+K_{4}(R, 0)
\end{array}\right),
$$

where, for example,

$$
K_{3}\left(R, k_{f}\right)=\int d^{3} s_{1} d^{3} s_{2} \psi^{\star}\left(k_{f}\right)\left[-\frac{d^{2}}{2 m_{q} d r_{3}^{2}}\right] \psi\left(k_{f}\right) .
$$

Again these integrals can be expressed in forms similar to that in Eq. 16.

Finally, the potential matrix has the form

$$
\mathbf{V}^{\prime}\left(R, k_{f}\right)=\left(\begin{array}{ll}
\langle v(13), 0\rangle+\langle v(24), 0\rangle & \left\langle V_{A B}, k_{f}\right\rangle \\
\left\langle V_{A B}, k_{f}\right\rangle & \langle v(14), 0\rangle+\langle v(23), 0\rangle
\end{array}\right),
$$

where

$$
\begin{gathered}
\left\langle V_{A B}, k_{f}\right\rangle= \\
\frac{1}{3}\left[\left\langle v(13), k_{f}\right\rangle+\left\langle v(24), k_{f}\right\rangle+\left\langle v(14), k_{f}\right\rangle+\left\langle v(23), k_{f}\right\rangle-\left\langle v(34), k_{f}\right\rangle-N\left(R, k_{f}\right) V(2, R)\right] .
\end{gathered}
$$

Here $N\left(R, k_{f}\right)$ is defined in Eq. 15, $V(2, R)$ is the potential between the two heavy quarks and, for example,

$$
\left\langle v(13), k_{f}\right\rangle=\int d^{3} s_{1} d^{3} s_{2} \psi^{\star}\left(k_{f}\right) V\left(s_{1}\right) \psi\left(k_{f}\right) .
$$


For potentials of the form in Eq. 月, these integrals can be expressed in terms of Error functions. The energy $E\left(4, k_{f}\right)$ of the two heavy-light meson system is then obtained by diagonalising

$$
\left|\mathbf{K}^{\prime}\left(R, k_{f}\right)+\mathbf{V}^{\prime}\left(R, k_{f}\right)-E\left(4, R, k_{f}\right) \mathbf{N}^{\prime}\left(R, k_{f}\right)\right| \psi=0 .
$$

Since this is a $2 \times 2$ determinant a prediction could also be made for an excited state $E^{*}\left(4, k_{f}\right)$ and the corresponding binding energy $B^{*}(4)$.

We saw that the variational method worked very well for the two-quark energies. In the four-quark case, when the intermeson interaction $\left\langle V_{A B}, k_{f}\right\rangle$ is set to zero (i.e. $k_{f} \rightarrow \infty$ - the strong coupling limit), a necessary condition is that $E\left(4, k_{f}=\infty\right)-2 E(2, L=0)$ should be approximately zero. This is found to be sufficiently well satisfied, provided $N_{4}$ in Eq. 12 is at least 2. There is a small remaining repulsion of about $5 \mathrm{MeV}$ due to inadequacies in the $\psi(r, f)$ of Eq. 12 and this could presumably be made smaller by improving this wavefunction. However, $5 \mathrm{MeV}$ should be compared with the two body energy of $709 \mathrm{MeV}$, which is made up from a kinetic energy of $339 \mathrm{MeV}$ and a potential energy of $370 \mathrm{MeV}$. So we see that the condition $E(4)=2 E(2)$ is satisfied to within $1 \%$.

\section{Results}

One of the main ingredients of the above model is the interquark potential $V(2, r)$. This enters in three different contexts:

1) As $v(13), v(24), v(14), v(23)$ - the $Q \bar{q}$ potential in Eq. 3 .

2) As $v(34)$ - a $\bar{q} \bar{q}$ potential. Here we assume this to also be of the form in Eq. 3

$3)$ As $v(12)$ - a $Q Q$ potential. This was calculated from the same gauge configurations as the four-quark energies. In this case there was no need to fit this with a function of $R$, since it is only ever needed at discrete values of $R$ - the ones for which the four-quark energies are calculated.

This prescription for $V(2, r)$ is the one throughout this article. However, to check the dependence of the following results on this choice, several other options were considered. But in all cases the same qualitative conclusions emerged.

Given these two-quark potentials, then the results from the four-quark model, described in the previous section, can now be compared with the lattice calculations of Ref. [11]. This model gives the binding energy of the four-quark state using a spin-isospin independent interaction. Therefore, in order to make a comparison with the lattice data of Ref. [11], which are dependent on the spin $\left(S_{q}\right)$ and isospin $\left(I_{q}\right)$ of the $(\bar{q} \bar{q})$ subsystem, an averaging of this data must be made. This averaging could be avoided if, in addition to the spin independent interaction of Eq. 3, the model also contained a spin dependent interaction. However, the later is expected to be of short range - in fact a delta-function from One Gluon Exchange - and so be less affected by any four-quark form factor. Therefore, by only considering the spin independent contributions 
to the binding, we hope to maximise the effect we are studying - namely the need for such a form factor. Of course, in further developments of the model this restriction will clearly need to be lifted and both the spin-independent and -dependent potentials included. To carry out the averaging we are guided by the weak coupling limit - the same limit already used in setting up the basic form of the model in Eqs. 1- 11. As shown in the Appendix of Ref. [18] the two basis states $A$ and $B$ have a color structure of the form:

$$
|A\rangle=|(1 \overline{3})(2 \overline{4})\rangle=\sqrt{\frac{1}{3}}|[12][\overline{3} \overline{4}]\rangle+\sqrt{\frac{2}{3}}|\{12\}\{\overline{3} \overline{4}\}\rangle
$$

and

$$
|B\rangle=|(1 \overline{4})(2 \overline{3})\rangle=-\sqrt{\frac{1}{3}}|[12][\overline{3} \overline{4}]\rangle+\sqrt{\frac{2}{3}}|\{12\}\{\overline{3} \overline{4}\}\rangle,
$$

where $(\ldots)$ denotes a color singlet, $[\ldots]$ a color triplet and $\{\ldots\}$ a color sextet. The overlap $\langle A \mid B\rangle$ gives the factor of $1 / 3$ appearing in Eq. 7 and also we have the relationship

$$
|A\rangle-|B\rangle=\frac{2}{\sqrt{3}}([12][\overline{3} \overline{4}])
$$

i.e. in this combination of $A$ and $B$ the $(\bar{q} \bar{q})$ subsystem is in a color triplet state and so antisymmetric in color. Since the lattice data only involves S-wave interactions, $\left(I_{q}, S_{q}\right)$ must be $(0,0)$ or $(1,1)$ to ensure overall antisymmetry for the interchange $\bar{q}_{3} \leftrightarrow \bar{q}_{4}$. For an interquark interaction of the form $V=V_{0}+\mathbf{s}\left(\overline{\mathbf{q}}_{\mathbf{3}}\right) \cdot \mathbf{s}\left(\overline{\mathbf{q}}_{4}\right) V_{s}$, we have $V(0,0)=V_{0}-3 V_{s} / 4$ and $V(1,1)=$ $V_{0}+V_{s} / 4$. Therefore, to extract the effect of $V_{0}$ - the spin independent part of the interaction - we need for $|A\rangle-|B\rangle$ the combination $V_{0}=[3 V(1,1)+V(0,0)] / 4$. However, for $R=0$ the two diagonal matrix elements in each of the matrices $\mathbf{N}^{\prime}\left(R, k_{f}\right), \mathbf{K}^{\prime}\left(R, k_{f}\right), \mathbf{V}^{\prime}\left(R, k_{f}\right)$ in Eqs. 15, 17, 19 are equal, so that from Eq. 22 the wavefunction of the ground state is, indeed, simply proportional to $\frac{1}{\sqrt{2}}[A-B]$. As $R$ increases the amplitude of $A$ then increases to 0.75 at $R=0.18 \mathrm{fm}$ and eventually to unity for $R \geq 0.5 \mathrm{fm}$. Therefore, for the small values of $R$ of interest here, the ground state wavefunction is automatically approximately proportional to $[12][\overline{3} \overline{4}]$ as in Eq.25. This suggests that the above method for extracting $V_{0}$ should be sufficiently accurate.

Figure 2 compares the lattice results for $V_{0}$ (with and without dynamical sea quarks) of Ref. [11] using the weak coupling model (i.e. $f=1$ in Eq. 12 or $k_{f}=0$ in Eq. 14). The model is the one designed for the lattice data in the quenched approximation i.e. using $V(2, R)$ of Eq. 3. The preliminary data of Ref. [12] that includes dynamical sea quarks is simply included to show that there are no dramatic changes in the binding energies. The quenched data from Ref. [11] used the lattice parameters $\beta=5.7, \kappa=0.14077, C_{\mathrm{SW}}=1.76$ giving a lattice spacing of $a=0.170 \mathrm{fm}$ and mass ratio $M_{\mathrm{PS}} / M_{\mathrm{V}}=0.65$, whereas the unquenched data [12] has $\beta=5.7, \kappa=0.1395, C_{\mathrm{SW}}=1.52$ and $a=0.142 \mathrm{fm}, M_{\mathrm{PS}} / M_{\mathrm{V}}=0.72$. Figure 2 clearly shows that for $R=0.18 \mathrm{fm}$ the model overestimates the binding by over a factor of three. For larger values of $R$ the relative error bars on the small energies are, at present, too large to make any conclusions. Unfortunately, the lattice data at the smallest values of $R$ could well still contain 
lattice artifacts that are not completely cancelled in the difference defining the binding energy in Eq. 1 - a point discussed later. However, when the factor $f$ is no longer unity, the model binding decreases considerably. This is shown in Figure 3 for $k_{f}=0.05,0.10,0.15$ and $m_{q}=400 \mathrm{MeV}$ with the optimal value $k_{f} \approx 0.10$ fitting the first lattice data point. Also for this value of $k_{f}$ the variation with $m_{q}$ is found to be small. It should be added that the lattice spacing $a$ differs slightly $(0.18 \mathrm{fm}$ vs $0.17 \mathrm{fm}$ ) between that used for $V(2, r)$ based on Ref. [9] and the four-quark data of Ref. [11]. This is due to a difference in the procedure for extracting $a$. We could have scaled the data to a common $a$. But this refinement is not necessary for the present stage of precision.

The form factor in Eq. 14 can also be used in Eq. 11 to fit the $Q^{4}$ lattice data in Figure 1. There it is seen with the dashed lines that $k_{f}=0.075$ gives a good fit to both the ground and excited states over the whole range of $R$ values considered. This value of $k_{f}$ is in good agreement with that needed in Figure $2-k_{f} \approx 0.10$.

In Figure 2 is also shown the corresponding binding energy when the 2-quark potential of Ref. [15] is used in the model (dashed line). The difference between these two gives an estimate of the range of values that can be expected using different 2-quark potentials. In all cases, the model overbinds by a large factor. The difference is also readily understandable by considering the small $R$ limit of the model. There it is seen that it is the $V(2, R)$ in Eq. 20 that dominates. This results in $E(4, R) \rightarrow V(2, R) / 2$ as $R \rightarrow 0$. If this limit were already reached at $R=1$, then we would expect - from the estimates of $V(2, R=1)$ given after Eq. 3 - that the binding energies from the two versions of $V(2, R)$ should be about $-70 \mathrm{MeV}$ and $-90 \mathrm{MeV}$. Eventhough these limits underestimate by almost a factor of two the model values in Figure 2, they have the correct trend and also emphasise the importance of needing a good model for $V(2, R)$.

The above comparisons between the four-quark lattice energies and the variational estimates have at least three shortcomings:

a) The most serious problem with the variational calculation is that $m_{q}$ is sufficiently small that a relativistic form for the kinetic energy should be used both in the two- and four-quark variational formulations for the energy. An indication of the effect of using the non-relativistic approximation can be estimated by comparing the effective two-body kinetic energy in the four quark case with the kinetic energy in the original two-body problem i.e. compare $K_{3}(R, 0)$ [or $K_{4}(R, 0)$ - they are equal] in Eq. 17 with $\langle\phi(r)|T(2)| \phi(r)\rangle$ in Eq. [4, which has the value 339 $\mathrm{MeV}$. It is found that $K_{3}(R, 0)$ is quite dependent on $k_{f}$ but much less so on $R$. For example, at $R=0.18 \mathrm{fm}$ and $k_{f}=0.0,0.15,0.25,0.45$ we find $K_{3}(R, 0)=286,328,338,346 \mathrm{MeV}$ respectively. Therefore, in the difference $B(4)=E(4)-2 E(2,0)$ much of the kinetic energy terms cancel - especially after the inclusion of the form factor needed to tune the model energy to the lattice value. In fact, for the optimal value of $k_{f} \approx 0.25$ the cancellation is within a few $\mathrm{MeV}$. For larger values of $R$ the cancellation is even more complete. This leads to the expectation (hope) that a similar cancellation will occur in a relativistic formulation and that 
the binding energies calculated with the present non-relativistic model are indeed quite realistic.

b) The second problem is that there is some ambiguity in the form of the two-quark potential $V(2, r)$ in Eq. 3, since it is a fit to lattice $Q \bar{q}$ potentials that are only known at discrete values of $r$. This fit was designed to ensure that $V(2, r)$ reproduced well the lattice potentials over the range $2 \leq r / a \leq 4$. The reason for this is found from Table 2, where it is shown how the effective two-body potential $(V T)$ is made up from the two potential components - linear $V L$ and coulomb-like $V C$ - in $V(2, r)$ of Eq. 3. These can be compared with the corresponding two-quark contributions, namely, 370, 524, $-154 \mathrm{MeV}$ respectively. If we now define $n$ as the ratio $-V L / V C$, then a rough estimate of the most important range of $r$ values for the integrals is given by

$$
r_{I} \approx a \sqrt{\frac{n e}{b}}
$$

where $e=0.309$ and $b=0.165$ from Eq. 3. In this case from Table 2 we have $n \approx 3$, so that $r_{I} \approx(2-3) a \approx(0.4-0.5) \mathrm{fm}$. At such values of $r$, lattice artifacts should be small. Therefore, Eq. 3 is expected to be valid and yield reliable values for the radial integrals. In Figure 2 was shown the dependence of the four-quark binding energy on the choice of 2-quark potential. There it was seen that the potential proposed in Ref. [15] gave even more overbinding than that in Eq. 3. In order to correct this, therefore, needs a stronger (i.e. shorter ranged) form factor with $k_{f} \approx 0.4$.

c) A third shortcoming in the above formulation is in the form of the variational wavefunction in Eq. 12. This could be improved in two ways. Firstly, the limitations to $b_{i}=0, d_{i}=a_{i}$ and $e_{i}=c_{i}$ in Eq. 13 for the form of the $\mathbf{M}_{\mathbf{i}}$ could be removed. This would presumably remove part of the $5 \mathrm{MeV}$ difference in the $E\left(4, k_{f}=\infty\right)-2 E(2, L=0)$ mentioned earlier. However, secondly, Eq. 12 is designed to describe states that are naturally in terms of the $\mathbf{s}_{\mathbf{i}}$ radial coordinates defined just before that equation. This means that this form is optimal for state $A$ but not state $B$, which is described naturally in terms of the $\mathbf{t}_{\mathbf{i}}$ radial coordinates. The error introduced by this can be roughly estimated by minimizing the energy of the excited state and treating the ground state model energy as a prediction. However, in practice, this seems to be unimportant for the values of $R$ of interest here. For example, in the strong coupling limit, when $k_{f}$ is very large (140 here), minimizing the ground state energy at $R=0.26 \mathrm{fm}$ leads to $B(4), B^{*}(4)$ having 4.8 and $108 \mathrm{MeV}$ respectively, whereas the corresponding numbers are 7.5 and $105 \mathrm{MeV}$ when it is the excited state that is targetted in the minimization. At smaller values of $R$ the differences are even smaller.

\section{Conclusions}

The main conclusion is most clearly demonstrated in Figure 2, where it is seen that the present model in the weak coupling limit overestimates the binding energy given by the lattice simulation. 
This means that the four quark systems $\left(Q^{2} \bar{q}^{2}\right)$ studied here cannot be described simply in terms of two-quark potentials. The effect of the latter needs to be suppressed and this is achieved here by introducing the explicit four-quark form factor $f$ shown in Eq. 14. By itself this conclusion would not be very convincing, since it all depends on the one lattice data point at $R / a=1$. However, when this result is combined with the more reliable, but less physically interesting case, of four static quarks from our earlier work, we see that the same trend is observed and that the combined result adds further support to our main conclusion. In fact this support is not only qualitative but also quantitative, since the optimal values for $k_{f}$ in Figures 1 and 3 are quite similar - being 0.075 for $Q^{4}$ and 0.10 for $Q^{2} \bar{q}^{2}$. Furthermore, the hope is that the form factor needed to tune the model to the lattice data is universal in the sense that it can be used in systems with more than four quarks. This is the ultimate aim of this work - a bridge between few quarks systems amenable to lattice calculations and multi-quark systems that are beyond such methods and so rely on more conventional many-body techniques.

In the future both the lattice simulation and the model will be improved. As mentioned above, the lattice data will eventually give binding energies with smaller error bars at larger values of $R$. This could be not only for the ground state but also for excited states. Also the effect of using unquenched light quark propagators is now becoming possible [12]. The model is also capable of being extended and improved in several ways. For example, in the $Q^{2} \bar{q}^{2}$ system it could be extended to include a spin dependence in the basic interaction of Eq. 3. Also the basic form of the variational wavefunction in Eq. 12 could be improved by using more of the parameters in the matrix $\mathbf{M}$ of Eq. 13. However, such an improvement would presumably lead to more binding and so increase even more the difference between the lattice data and the basic two-quark potential model without the four-quark form factor. Of course, the point needing most improvement is the treatment of the kinetic energy, eventhough the indications are that the kinetic energy in the four- and two-body systems to a large extent cancel each other. At present, only the non-relativistic form is used. However, it should be possible to treat a semirelativistic version of the model, where $m_{q}+p^{2} / 2 m_{q}$ is simply replaced by $\sqrt{p^{2}+m_{q}^{2}}$ and the kinetic energy integrals performed in momentum space. Unfortunately, this will result in a formulation that involves a one-dimensional numerical integration over momentum.

Another improvement that could become necessary with improved data would be the addition of new basis states with excited two-body potentials. The excited states were found necessary in our previous fit to a large set of precisely measured energies in the static $\mathrm{SU}(2)$ case, with the main conclusion about these states being that they have a larger effect than the ground state potentials on the binding for large $R$ (beyond $0.5 \mathrm{fm}$ ). However, in the present more dynamic system an additional effect for $R \geq 0.5 \mathrm{fm}$ would come from meson exchange, for which impressive agreement in this distance range was found in Ref. [11]. A Yukawa potential effective at long distances could be added to relevant matrix elements of our model in order to reproduce the observed deuson-like behavior [19]. Such a potential is expected to be essential for the observed binding of $\left(I_{q}, S_{q}\right)=(0,1) ;(1,0)$ states not discussed in the present work. 
The authors wish to thank Ted Barnes, Tim Klassen, Chris Michael and Jean-Marc Richard for useful discussions and correspondence. We are also grateful to the CSC in Espoo for their excellent computational resources and to the Finnish Academy for supporting this project.

\section{References}

[1] T. Barnes, N. Black, D.J. Dean and E.S. Swanson,'BB Intermeson Potentials in the Quark Model', nucl-th/9902068

S. Pepin, F. Stancu , M. Genovese and J.M. Richard, Phys. Lett. B393, 119 (1997)

[2] T. Barnes, S. Capstick, M.D. Kovarik and E.S. Swanson, Phys. Rev. C 48, 539 (1993)

[3] A.M. Green, J. Lukkarinen, P. Pennanen, C. Michael, Phys. Rev. D 53, 261 (1996), hep-lat/9508002; P. Pennanen, Phys. Rev. D 55, 3958 (1997), hep-lat/9608147.

[4] A.M. Green and P. Pennanen, Phys. Rev. C 57, 3384 (1998), hep-lat/9804003.

[5] A.M. Green, C. Michael and J. Paton, Nucl.Phys. A554, 701 (1993).

[6] M.B. Gavela et al., Phys. Lett. B82, 431 (1979)

[7] M. Oka and Yazaki, "Quarks and Nuclei", Int. Review of Nuclear Physics - Vol. 1 (1984) p.489, eds.T.T.S.Kuo and E.Osnes (World Scientific Publishing Co. Singapore 1984)

[8] F. Lenz et al., Ann. of Phys. 170, 65 (1986)

[9] C. Michael and J. Peisa, Phys. Rev. D 58 034506-1 (1998), hep-lat/9802015.

[10] C. Michael, Proc. of the CONFINEMENT III conference in Newport News (1998), hep-ph/9809211; P. Pennanen, A.M. Green and C. Michael, Proc. of LATTICE98, Boulder (1998), hep-lat/9809035.

[11] C. Michael and P. Pennanen, NORDITA-98/79, LTH-449, hep-lat/9901007.

[12] UKQCD Collaboration C. Michael and P. Pennanen, work in progress.

[13] UKQCD Collaboration C.R. Allton et al., 'Light hadron spectroscopy with $O(a)$ improved dymamical fermions', hep-lat/9808016

[14] J. Izen, BaBar Hadronic b/c Physics at SLAC - April 14th 1999 and private communication.

[15] R.G. Edwards, U.M. Heller and T.T. Klassen, Nucl. Phys. B517, 377 (1998), hep-lat/9711003.

[16] B. Masud, J. Paton, A.M. Green and G.Q. Liu, Nucl.Phys. A528, 477 (1991). 
[17] E. Eichten, K. Gottfried, T. Kinoshita, K.D. Lane and T.-M. Yan, Phys.Rev. D 17, 3090 (1978); 21, 313 (E)(1980).

[18] J. Weinstein and N. Isgur, Phys. Rev. D 41, 2236 (1990).

[19] N. Törnqvist, Z.Phys. C61, 525 (1994), hep-ph/9310247. 


\section{Figure Captions}

Figure 1. The case of four static quarks $\left(Q^{4}\right)$ in $\mathrm{SU}(2)$ placed at the corners of squares of side $R / a$, where $a \approx 0.12 \mathrm{fm}$. This shows the binding energy $(\mathrm{aB})$ in lattice units as a function of $R / a$. The lattice results are for $\beta=2.4$ on a $16^{3} \times 32$ lattice with the dots(crosses) showing the ground(excited) state energies - with error bars. The lower(upper) solid curve is a model estimate for the ground(excited) binding energy obtained assuming only 2-quark interactions. This figure is similar to the one in [5]. The lower(upper) dashed curve is the effect of the form factor in Eq. 14 with $k_{f}=0.075$.

Figure 2. Comparison between the spin independent part $\left(V_{0}\right)$ of the $Q^{2} \bar{q}^{2}$ binding energies calculated on a lattice [11] (solid circles - quenched approximation with $a=0.170 \mathrm{fm}$ )/ [12] (solid squares - with dynamical fermions and $a=0.142 \mathrm{fm}$ ) and the model in the weak coupling limit $\left(k_{f}=0\right)$. The crosses, with the solid line to guide the eye, use the two-quark potential in Eq. 3 and the stars, with the dashed line, the two-quark potential in Ref. 15. The dynamical fermion data is not used in any fit. It is simply included to show that it is qualitatively consistent with the quenched data but with considerably smaller error bars.

Figure 3. Comparison between the spin independent part $\left(V_{0}\right)$ of the $Q^{2} \bar{q}^{2}$ binding energies calculated on a lattice [11] and using the variational principle with $k_{f}=0.15$ (dotted), 0.10 (solid), 0.05 (dashed) and $m_{q}=400 \mathrm{MeV}$. Other notation as in Figure 2. 
Table 1: The comparison, as a function of $r / a$, between the two-quark potential $a V(2, r)$ of Eq. 3 with the additive constant removed and the potential given by Wilson loops in Ref. [11.

\begin{tabular}{cccccc}
\hline$r / a$ & 1 & 2 & 3 & 4 & 5 \\
\hline \hline$a V(2, r)$ & -0.144 & 0.175 & 0.392 & 0.582 & 0.763 \\
11] & $-0.126(1)$ & $0.175(2)$ & $0.392(3)$ & $0.586(9)$ & $0.783(22)$
\end{tabular}

Table 2: The effective two-body potential $(V T)$ in $\mathrm{MeV}$ for the four-quark system at $R=0.18$ fm and $k_{f}=0.25$. The $V L$ and $V C$ are the linear and coulomb-like contributions to $V T$.

\begin{tabular}{c|ccc} 
& $V L$ & $V C$ & $V T$ \\
\hline$\left\langle V\left(\mathbf{s}_{\mathbf{1}}\right)\right\rangle$ & 300 & -102 & 198 \\
$\left\langle V\left(\mathbf{t}_{\mathbf{1}}\right)\right\rangle$ & 315 & -97 & 218 \\
$\langle V(\mathbf{u})\rangle$ & 423 & -72 & 351
\end{tabular}




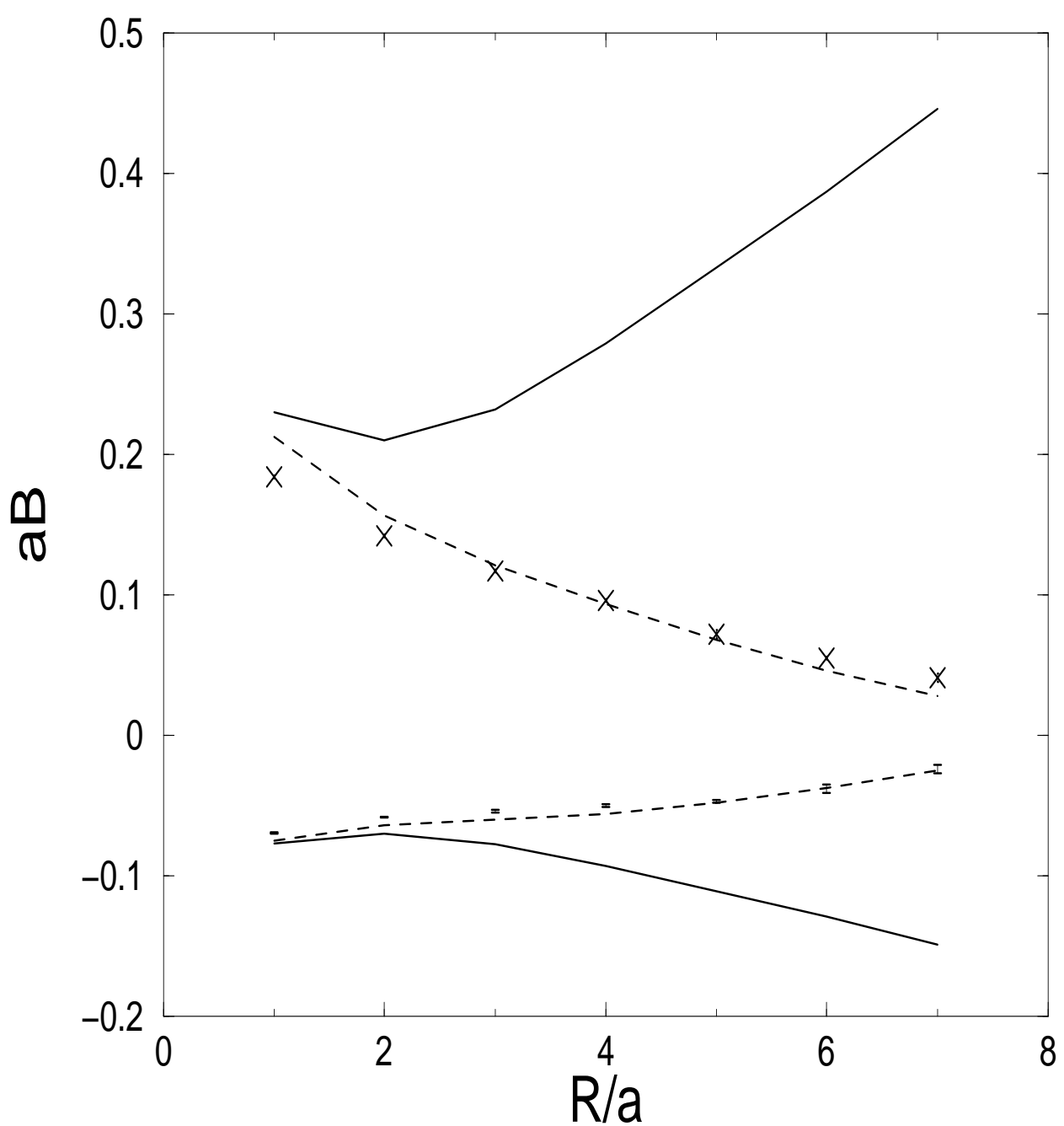

Figure 1: The case of four static quarks $\left(Q^{4}\right)$ in $\mathrm{SU}(2)$ placed at the corners of squares of side $R / a$, where $a \approx 0.12 \mathrm{fm}$. This shows the binding energy $(\mathrm{aB})$ in lattice units as a function of $R / a$. The lattice results are for $\beta=2.4$ on a $16^{3} \times 32$ lattice with the dots(crosses) showing the ground(excited) state energies - with error bars. The lower(upper) solid curve is a model estimate for the ground(excited) binding energy obtained assuming only 2-quark interactions. This figure is similar to the one in [5]. The lower(upper) dashed curve is the effect of the form factor in Eq. 14 with $k_{f}=0.075$. 


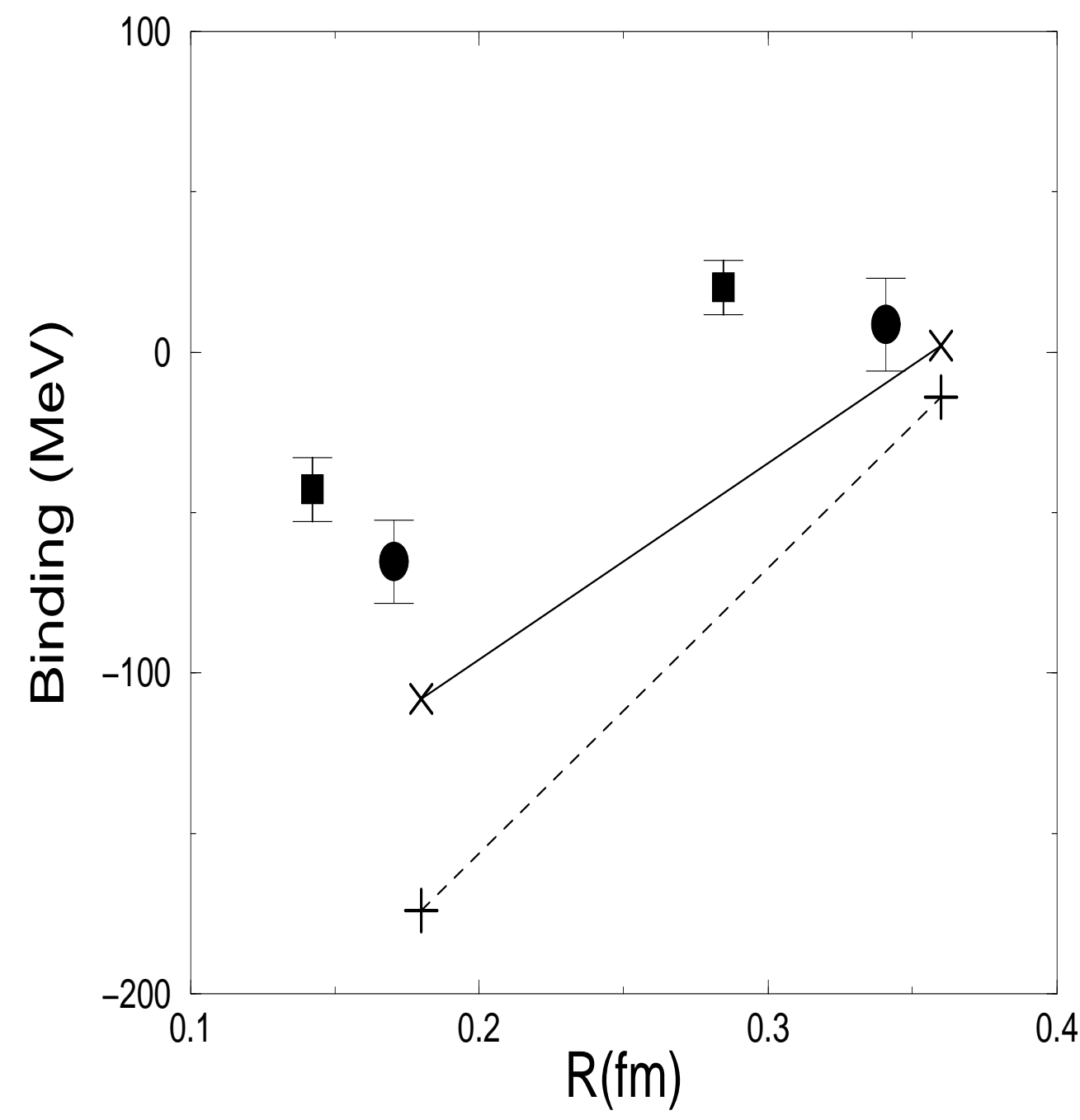

Figure 2: Comparison between the spin independent part $\left(V_{0}\right)$ of the $Q^{2} \bar{q}^{2}$ binding energies calculated on a lattice [11] (solid circles - quenched approximation with $a=0.170 \mathrm{fm}$ )/ [12] (solid squares - with dynamical fermions and $a=0.142 \mathrm{fm}$ ) and the model in the weak coupling limit $\left(k_{f}=0\right)$. The crosses, with the solid line to guide the eye, use the two-quark potential in Eq. 3 and the stars, with the dashed line, the two-quark potential in Ref. 15. The dynamical fermion data is not used in any fit. It is simply included to show that it is qualitatively consistent with the quenched data but with considerably smaller error bars. 


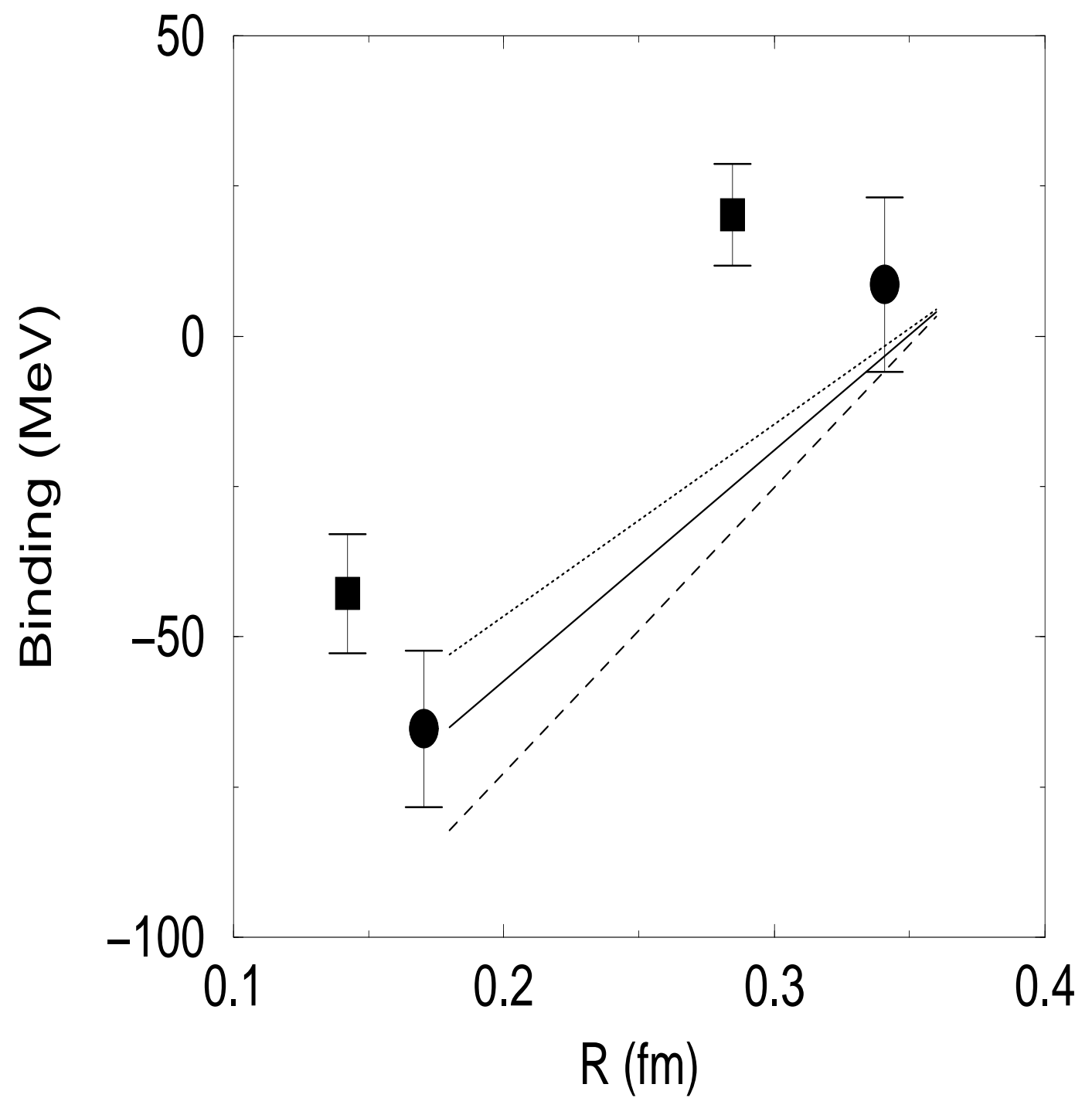

Figure 3: Comparison between the spin independent part $\left(V_{0}\right)$ of the $Q^{2} \bar{q}^{2}$ binding energies calculated on a lattice [11] and using the variational principle with $k_{f}=0.15$ (dotted), 0.10 (solid), 0.05 (dashed) and $m_{q}=400 \mathrm{MeV}$. Other notation as in Figure 2. 